\title{
Physiopathology of Pulmonary Airways: Automated Facilities for Accurate Assessment
}

\author{
Diane Perchet, Catalin I. Fetita, and Françoise Prêteux \\ ARTEMIS Project Unit, INT, Groupe des Ecoles des Télécommunications \\ 9 rue Charles Fourier, 91011 Evry Cedex, France
}

\begin{abstract}
In the framework of computer-assisted diagnosis, pulmonary airway investigation based on multi detector computed tomography $(\mathrm{MDCT})$ requires to provide radiologists and clinicians with advanced tools for interactive exploration, quantitative assessment and follow-up. This paper develops a set of automated investigation facilities relying on 3D airway reconstruction, enhanced central axis-based description and accurate meshing. By overcoming the limitations encountered by the current post-processing techniques available in clinical routine, the proposed tools contribute to increase the diagnosis confidence level and provide better insights into the physiopathology of airways.
\end{abstract}

\section{Introduction}

Providing radiologist with non-invasive facilities for airway pathology diagnosis and follow-up is still a challenging issue for the medical imaging industry. With the development of multi-detector computed tomography (MDCT), a high resolution imaging of the thorax in a single breath hold is now possible, allowing to assess focal or diffuse, proximal or distal airway diseases [1]. However, an accurate quantitative investigation of the physiopathology of pulmonary airways requires advanced investigation tools which are not available in clinical routine yet. Such tools mainly involve the access to the $3 \mathrm{D}$ information on the tracheobronchial tree carried by the MDCT acquisition. In this way, several local analyses become possible: evaluation of the extent of stenosis and bronchiectasis for treatment planning and follow-up, assessment of complex airway abnormalities, study of the airway remodeling in the case of chronic obstructive pulmonary disease (COPD) and asthma, etc.

Starting from an accurate and robust 3D reconstruction of the bronchial tree from MDCT acquisitions, this paper develops additional facilities for local analysis, thus providing new insights into the physiopathology of airways. After an overview of the demanded tools in clinical routine and of the current limitations of the existing techniques $(\S 2)$, an unified solution relying on a multivalued central axis-based representation is proposed ( $\$ 3)$. The resulting clinical applications are illustrated and discussed in $\S 4$. 


\section{Morphofunctional Analysis of Airways: Medical Desiderata and Technical Requirements}

The 3D reconstruction of airways from MDCT becomes critical to a full assessment of their physiopathology. In a previous work 2 we have developed a fullyautomated 3D segmentation approach which combines a strong morphological filtering (in order to extract a low-order airway subset), and an energy-based aggregation model (able to reconstruct high-order bronchi). The bronchial tree is reconstructed up to the $6^{t h}-7^{t h}$ subdivision order, even in the presence of local obstructive pathologies. However, at this stage, such a $3 \mathrm{D}$ reconstruction only allows a global analysis of the airways. Additional tools are required for local investigation and quantification: lumen/wall cross-sectional area estimation, surgical planning and analysis via virtual bronchoscopy (VB), computational fluid dynamics (CFD). In this respect, some particular technical aspects have to be considered: (1) local estimation of the bronchus orientation, (2) virtual camera centrality problem in VB automatic navigation, (3) automated and accurate surface mesh generation from the binary 3D reconstruction for VB-based analysis and CFD. While the first two requirements find a solution with the central axis computation, the third one raises several difficulties related to the high branching complexity and to the caliber variability of the airway structure.

The existing algorithms performing the extraction of an object surface mesh from volumetric data can be classified into the following categories: planar contour-based methods [3], deformable models [4,5], and the "Marching Cubes" (MC) algorithm [6.7]. Widely used in medical imaging [8], the MC are better adapted to the bronchial tree structure. However, the MC-based surface meshes engendered directly from the 3D binary segmented data reveals strong irregularities corresponding to the voxel contours as well as some discontinuities in the small caliber branches, Fig. 1.

In order to prevent such effects, the commonly used approach consists in smoothing the binary data by means of a Gaussian filtering prior to applying the MC [8]. The surface mesh smoothness directly depends on the considered filter size. In the case of segmented airways, a large filter size induces geometrical distortions (segment shortening, diameter increasing and branch discontinuities) at the level of small caliber segments, Fig. 2 while a small filter size preserves the surface irregularities for large caliber bronchi. In addition, in the case of pathological configurations, abnormal mesh irregularities may also appear for large caliber segments and large filter sizes. Fig. 3 shows an ectopic bronchus originated from the trachea and generating a tracheal stenosis. The problem results from the subdivision morphology which keeps the segments very close one to another after the bifurcation. When a Gaussian smoothing is applied to such a configuration, local gray level maxima appear near the bifurcation. They may belong to the value range used for MC isosurface generation, which results in "spikes" on the 3D mesh, Fig. 3 .

In order to overcome these limitations and to generate a smooth and accurate 3D surface mesh of airways, two requirements are imposed: an adaptive data smoothing according to the bronchus caliber, on the one hand, and an adaptive 
control of the isosurface values, on the other hand. Such procedure can rely on the information provided by the central axis concerning geometry, topology, segment indexation and local caliber. Hence, central axis computation appears as the key issue in providing local airway investigation and quantification facilities. This issue will be addressed in the following section.

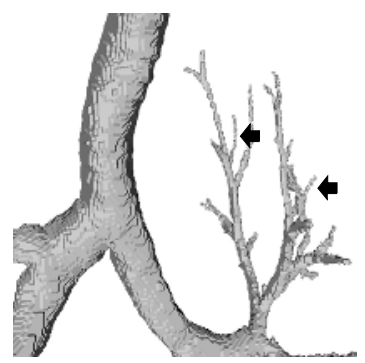

Fig. 1. MC applied to the binary data: irregular surface mesh and local discontinuities at the level of small caliber segments (arrows)

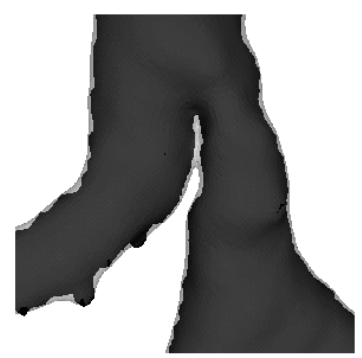

(a) Lateral view. The smoothed surface is displayed in light gray, superimposed on the nonfiltered surface

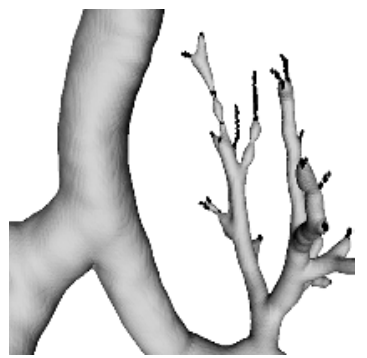

Fig. 2. Smoothed surface mesh by Gaussian filtering prior to MC (light grey) superimposed on the surface mesh from Fig 1 (black)

Fig. 3. Mesh generation with Gaussian pre-filtering for a pathological configuration

\section{Central Axis Computation}

Central axis (CA) algorithms can be classified into three main categories: thinning algorithms, methods based on Voronoi diagram computation and methods using distance maps.

The thinning algorithms iteratively "peel off" the object boundary, layer by layer, until one central layer remains. In $3 \mathrm{D}$, the most challenging issue is the 
connectivity preservation and such methods fail in preserving both connectivity and geometry, especially in the case of highly branching objects [11.

The second class of methods uses the property that the median axis of a polygon is a subset of its Voronoi diagram [12] 13. Rather adapted to polyhedrondefined objects, these algorithms present limitations in the case of noisy boundary objects: the Voronoi diagram thus obtained is highly complex and therefore imposes a severe skeleton pruning.

The distance map-based algorithms generally use a geodesic distance map or a geodesic front propagation with respect to a source point, and build the central axis by linking the centroids of the successive fronts. To ensure the centrality of the axis, many authors also refer to a distance map with respect to the object boundary in the computation of the centroids. The subdivision of a current front indicates that a branching point has been reached. The algorithm is then recursively applied to each subset of the splited front [14, Fig. 4(a) Since the 3D reconstruction of the bronchial tree reveals a quasi-tubular object with complex branching topology, this latter approach is better adapted to such a structure and was selected for our purpose.

However, the accurate detection of the branch subdivision points remains a key issue. Indeed, the usual criterion based on front splitting is not robust in the case of high caliber bronchi, and may lead to CA geometry distortions and hierarchy errors with respect to the bronchial subdivision order Fig. 4(b) 4(c)

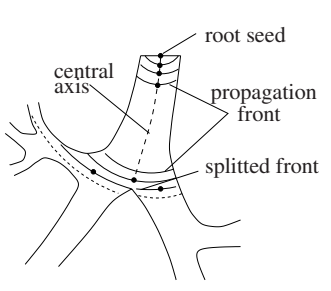

(a) Principle

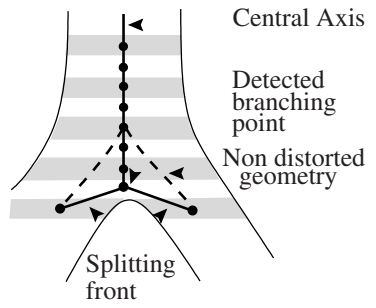

(b) Geometry distortion

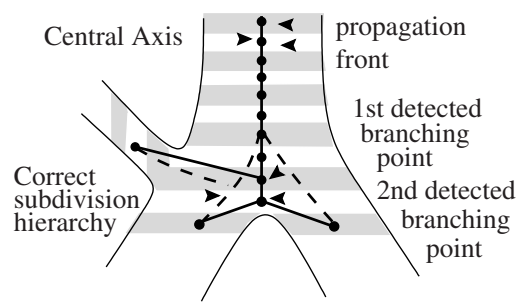

(c) Hierarchy errors

Fig. 4. CA extraction using geodesic front propagation and typical limitations due to branch subdivision detection based on front splitting

\subsection{The Developed Approach}

In order to overcome these limitations, we propose a robust criterion for subdivision point detection which combines geodesic front propagation and 3D distance map computed with respect to the bronchial wall.

Geometry distortion can be prevented by analyzing the 3D distance map information: the highest value points on the map correspond to the CA location. Thus, the occurence of a branching point can be suspected when several distance map local maxima appear at the level of the current propagation front. Such a technique prevents CA from geometry distortions, Fig. 5(a) but is insufficient 
to provide the correct configuration of a complex CA subdivision, Fig. 5(b) To achieve the correct hierarchy of the CA subdivision points, we propose a robust criterion based on a space partitioning starting from a possible subdivision point, detected as previously. First, the space partitioning defines the maximum sphere centered at this point and inscribed in the airway set. Then, the points located on the sphere surface propagate toward lower values of the distance map. This will generate a cone-shaped structure associated with each segment of the subdivision, irrespective of the degree of the subdivision (bifurcation, trifurcation, etc.). The geometry of the $\mathrm{CA}$ at the subdivision level is then reconstructed by linking the vertex of each cone-shaped structure to the subdivision point, by following the maximum value path on the distance map, Fig. 5(c). The advantage of such a space partitioning is to provide a global information on a subdivision area making it possible to manage any complex geometry.

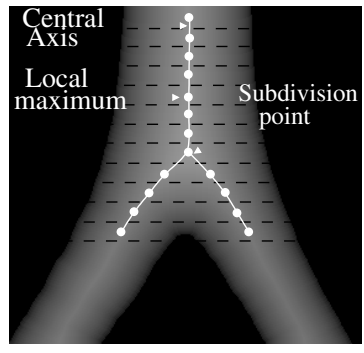

(a) Geometry correction

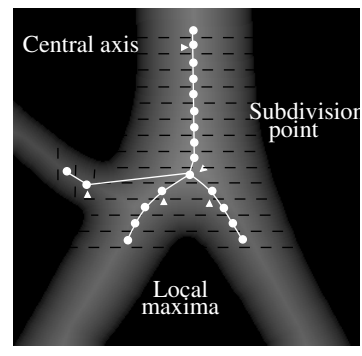

(b) Hierarchy errors

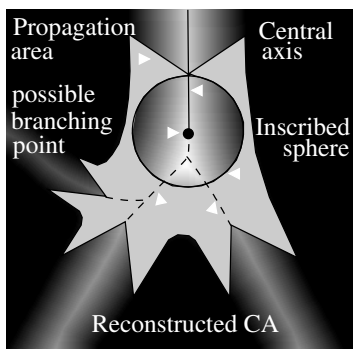

(c) Space partitioning

Fig. 5. Robust branching point detection with CA hierarchy preservation

The central axis is built up as a hierarchic and multi-valued tree structure: each node carries data related to the geometry, the topology, the caliber and the local orientation of the bronchus at this point.

\subsection{Accurate 3D Surface Mesh Construction}

Based on the $\mathrm{CA}$ information and on the MC algorithm, we propose a simple and efficient approach for accurate surface mesh construction from the binary $3 \mathrm{D}$ data. Such an approach consists in implementing an adaptive filtering of the binary reconstructed airways according to the bronchus caliber, and in locally controlling the isosurface values considered by the MC. The adaptive filtering aims at ensuring a constant diameter increasing ratio along the whole bronchial structure. Indeed, as shown in $§ 2$, large and small caliber bronchi are differently affected by a same (large size kernel) filter. The idea is to locally adapt the size of the Gaussian kernel to the bronchus caliber, in order to achieve the same diameter increasing ratio of the meshed bronchi with respect to the binary structure. Such an adaptation requires the identification of each bronchial segment, followed by a specific labeling which encodes the segment average radius. In this 
respect, the bronchial tree volume is divided in disjoint segments by isolating the influence region of each branch of the CA. During the filtering, the smoothed value of each voxel is computed by selecting the kernel size indicated in a lookup table with respect to the bronchus diameter. The look-up table was built up according to simulations carried out on a set of artificial tubular models which diameters vary in the same range as the physiological bronchi. For each model, these simulations determine which kernel size causes a relative radius increasing of $10 \%$ (the minimum value able to affect all generations of bronchi), and assign this value to the corresponding bronchus diameter in the table.

In order to avoid mesh irregularities due to pathologies, as shown in Fig. 3(b) the isosurface values resulting from the adaptive filtering should be locally controlled. In this respect, a second look-up table was empirically built up from the set of models previously described. The isosurface value assigned to each diameter is the gray-level value resulting from the adaptive filtering, at the level of the model initial contour. Fig. 6 shows the results provided by the method we proposed. The accuracy of the meshes thus generated has been validated by a radiologist expert who analyzed the mesh contours superimposed on bronchi cross sectional images at different subdivision orders.

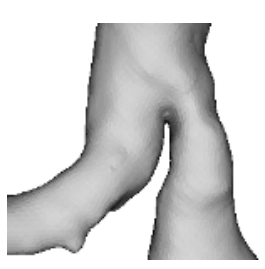

(a) External view

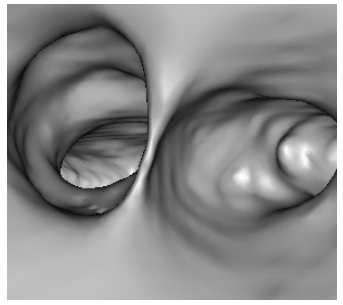

(b) Endoluminal view

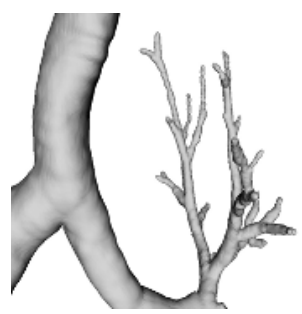

(a) Adaptive filter

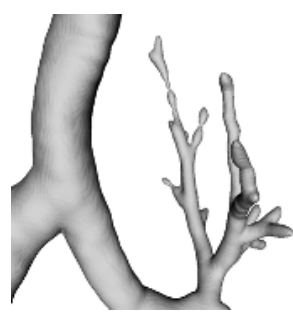

(b) Basic Gaussian

Fig. 7. Result of the adaptive filtering on small caliber bronchi

Fig. 6. Result of the adaptive filtering on the pathological configuration from Fig. 3

small caliber bronchi

\section{Insights into the Physiopathology of Pulmonary Airways}

The combination of the multi-valued information provided by the CA and the accurate reconstruction of the internal airway wall surface enables the development of facilities for local investigation, quantitative assessment, diagnosis and follow-up.

Morphometric information is automatically extracted from the CA structure (branching angles, segment length, Fig. 8). Similarly, the extent of obstructive pathologies, stenosis and bronchiectasis is easily estimated. A particular interest is presented by the accurate evaluation of the bronchial lumen wall surface, orthogonally to the bronchus axis, for the assessment of airway remodeling in 

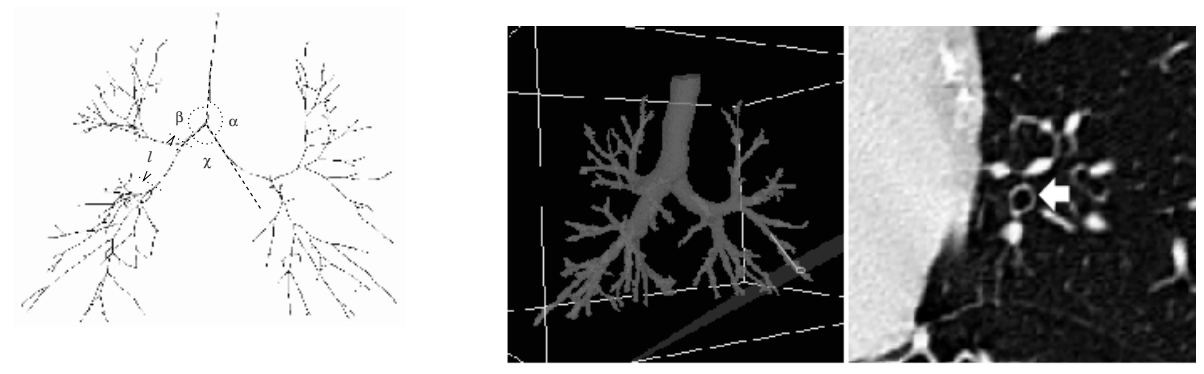

Fig. 8. Morphometric analysis

Fig. 9. Analysis of bronchial wall remodeling

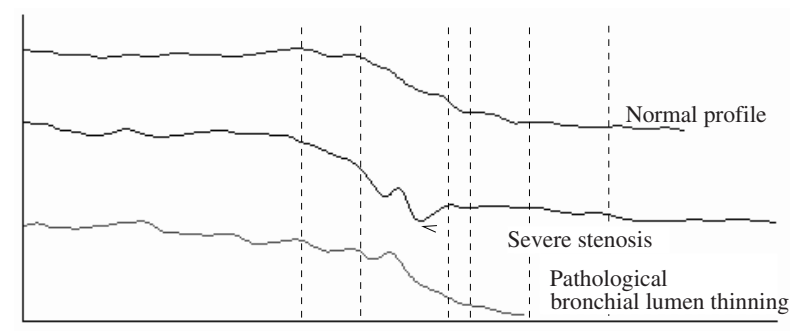

Fig. 10. Radius profiles from trachea down to a terminal segment

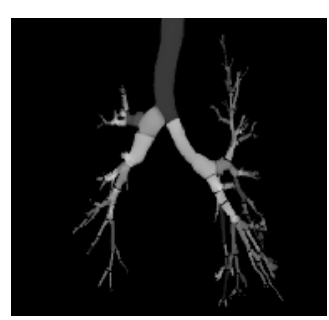

Fig. 11. Bronchial segment indexation

COPD and asthma. By using the CA information, cross-sectional multiplanar reformations (MPR) are possible at the same location, Fig. 9 , thus allowing airway comparison pre- and post-intervention (bronchoprovocation, bronchodilatation, therapeutic response).

Investigation paths along the CA are automatically computed starting from the trachea, down to the terminal segment, and bronchus caliber profile displayed with respect to a normal tree profile. Such plots give a first-hand information on the presence of obstructive pathologies, Fig. 10 An automatic procedure for bronchial segment indexation, Fig. [11, makes it possible to perform surgical planning and post-interventional follow-up. With the developments we proposed, airway analysis within virtual bronchoscopy becomes accurate for obstructive pathologies, Fig. 12, and for small caliber distal segments. CA provides automated trajectory computation between two selected points and ensures the centrality of the virtual camera inside the airway structure for an optimal investigation.

The accuracy of the reconstructed surface mesh enables to perform airflow simulations in realistic normal and pathological morphologies, with direct application to inhaled drug delivery [15]. 

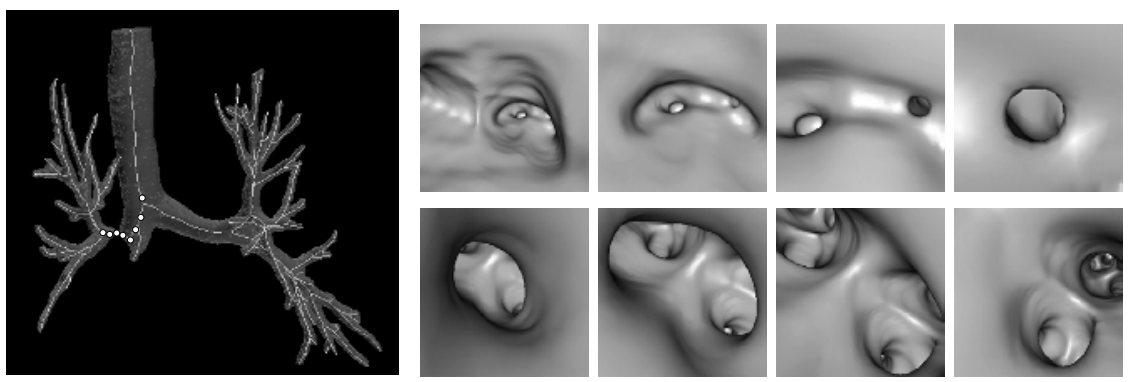

Fig. 12. Virtual bronchoscopy of a severe stenosis in the right upper lobe (RUL) bronchus. The navigation is performed from the right main bronchus down to the RUL bronchus (circles)

\section{Conclusion}

We have addressed the issue of the MDCT-based investigation of pulmonary airways in a clinical framework. Our objectives were to overcome the limitations encountered by the non-invasive exploration techniques currently available to the medical research and to provide radiologists with complementary tools for an accurate assessment of airway physiopathology.

The developed investigation facilities rely on a fully-automated 3D reconstruction of the bronchial tree from MDCT acquisitions and involve a hierarchic, multi-valued description and an accurate meshing of the complex airway structure derived from robust central axis computation. The resulting exploration tools make it possible to assess a large range of airway pathologies, to increase the diagnostic confidence level and to perform targeted and reproducible quantitative analysis.

Acknowledgement. This study was supported by the French National Project R-MOD.

\section{References}

1. Grenier, P., Beigelman, C.: Spiral CT of the bronchial tree. in Medical Radiology: Spiral CT of the Chest, M. Rémy-Jardin, J. Rémy eds., Berlin, Springer Verlag, (1996) 185-199.

2. Fetita, C. I., Prêteux, F., Beigelman-Aubry, C., Grenier, P.: Pulmonary airways: $3 \mathrm{D}$ reconstruction from multi-slice $\mathrm{CT}$ and clinical investigation. to appear in IEEE Trans on Med Imaging (2004).

3. Kwon, G.H., Chae, S.W., Lee, L.J.: Automatic generation of tetrahedral meshes from medical images. Computers and structures, Vol. 81 (2003) 765-775

4. McInerney, T., Terzopoulos, D.: Deformable models in medical image analysis: a survey. Medical Image Analysis, Vol. 1(2) (1996) 91-108 
5. Lachaud, J.-O, Montanvert, A.: Deformable meshes with automated topology changes for coarse-to-fine three-dimensional surface extraction. Medical Image Analysis, 3(2) (1998) 187-207

6. Lorensen, W.E., Cline, H.E.: Marching Cubes: a high resolution 3D surface construction algorithm. Computer Graphics, Vol. 21 (1987) 163-169

7. Wood, Z.J., Schroder, P., Breen, D., Desbrun, M.: Semi-Regular Mesh Extraction From Volumes. in Proc. of IEEE Visualization, (2000) 275-282

8. Summers, R.M., Cebral, J.R.: Tracheal and Central Bronchial Aerodynamics Using Virtual Bronchoscopy. in Medical Imaging 2001: Physiology and Function from Multidimensional Images, Chen, C-T and Clough, AV, Eds., Proceedings of SPIE Vol. 4321,(2001) 22-31

9. Lee, T.-Y., Lin, C.-H.: Growing-cube isosurface extraction algorithm for medical volume data. Computerized Medical Imaging and Graphics, Vol. 25 (2001) 405-415

10. Eck, M., Hoppe, H.: Automatic reconstruction of B-Spline surfaces of arbitrary topological type. ACM SIGGRAPH Proc., (1996) 325-334

11. Ma, C.M., Sonka, M.: A Fully Parallel 3D Thinning Algorithm and its Applications. Computer vision and image understanding, Vol. 64(3) (1996) 420-433

12. Kirkpatrick, D.G.: Efficient computation of continuous skeletons. IEEE 20th annual symphosium on foundations of computer science, (1979) 18-27.

13. Sherbrooke, E.C., Patrikalakis, N.M., Brisson, E.: An algorithm for the medial axis transform of 3D polyedral solids. IEEE transactions on visualization and computer graphics, Vol. 2(1) (1996) 44-61

14. Zhou, Y., Toga, A.: Efficient skeletonization of volumetric objects. IEEE Trans on visualization and computer graphics, Vol. 5(3) (1999) 196-209

15. Vial, L., Perchet, D., Fodil, R., Caillibotte et al: Airflow modeling in a CT-scanned human airway geometry. to appear in European Society of Biomechanics (ESB) Conference, (2004) 